\title{
Performance Evaluation of Wireless Sensor Network for Spatial and Aeronautic Systems
}

\author{
Akram HAKIRI $^{1,2}$, Pascal BERTHOU ${ }^{1,2}$, Julien HENAUT ${ }^{1,2}$, Daniela DRAGOMIRESCU ${ }^{1,2}$, Thierry GAYRAUD ${ }^{1,2}$ \\ ${ }^{1}$ CNRS ; LAAS-CNRS, 7, avenue du Colonel Roche, 31077 Toulouse, France \\ ${ }^{2}$ Université Toulouse; UPS, INSA, INP, ISAE; LAAS; F-31077 Toulouse, France \\ Email: \{Hakiri, Berthou, jhenaut, Daniela, Gayraud\}@laas.fr
}

\begin{abstract}
Ultra-wideband (UWB) has been proposed for physical layer standard for high speed wireless personal area networks (WPAN). It has emerged as cost effective and reliable alternative to traditional wireless technologies in WPANs.

This paper describes low complexity high data rate Ultrawideband system based on Wimedia communication protocol. It intends to evaluate the performance of Wimedia-UWB wireless networks communication for spatial and aeronautic systems. The paper suggests a simulation model using NS-2 simulator to assess the feasibility of such networks and analyses it performance by providing high data-rate wireless communication. Experimentations results are done using Wimedia-UWB toolkit to reproduce theses simulations conditions on Airbus wings and EADS-ASTRIUM satellite.
\end{abstract}

A simulation result shows the importance of the simulation approach to illustrate the satisfaction of the communication performance requirements and helps to reproduce simulation conditions in Hardware Test-Beds. Measurements prove the possibility of using such new approaches in aerospace industry.

\section{INTRODUCTION}

To evaluate sensor networks performance in spatial and aeronautic industry, simulation needs to be used to access the specific requirements of this industry. All of systems used today in aircraft and satellite industry are wired systems. Sensors put around the wings and satellite mockups are wired. The main drawbacks of this wired architecture are the high cost of cables and the difficulties encountered during the maintenance, unable to move the strands of cables needed for the instrumentation and increasing the availability related to faults associated with wiring (cutting, shearing, false contacts).

Indeed, Ultra Wideband [1] impulse radio based communication systems has known interest for last decade. Through the industry and the standardization community several activities aims to enable the deployment of this technology. Wimedia [2] MAC sub-layer was designed to support several different styles of wireless communication simultaneously. The price, the high data rate and the low power consumption of the Wimedia-UWB are the main motivator of using this technology.
Therefore, we consider a low complexity system for high data rate communication Wimedia-UWB system to design a wireless field bus for aerospace industry. These points let us describing a Wimedia-UWB wireless sensor network instrumentations field bus to simplify aeronautics flight tests to be used in Airbus planes and EADS-ASTRIUM satellites. Then, the simulation aims to evaluate the performance of such communication systems. Measurement experiences propagate the simulation conditions in hardware system to evaluate the performance of the system communication of the aircraft and validate its design.

The system is not completely designed, but a proof of concept was done using the Wimedia "off the shelves" communication systems. The NS-2 Wimedia model we developed allowed us to validate the compliance of the application constraints with this protocol. Wimedia UWB technology is a viable solution to enable high throughput data delivery in wireless networks. By the use of 'MAS', Wimedia allows several devices to cooperate with each other to share the medium access without requiring any master of the wireless bus to coordinate their transmission. This approach lets Wimedia support a high data rate communication up to $480 \mathrm{Mbps}$, and can be expected to move to gigabit data rates in the future.

This paper is organized into seven sections: after a brief introduction, section II introduces the motivation and the reasons of the choice of the Ultra Wideband technology to support the SACER project. An overview of the WimediaUWB protocol stack is given in section III. We give in section IV a description of the models we developed. The simulation results and the performance analyses are shown in section VI. Section VII shows tests and measurements performed with real electronic devices indoor and inside a mockup. Finally, conclusions are drawn in the last section.

\section{SCOPE AND MOTIVATION}

The context of the work takes place in wireless sensor networks communication project called SACER. In the SACER project (figure 1) a wireless instrumentation field bus is being designed to simplify aeronautics flight tests.

Thus, like shown in figure 1 , the architecture aims to produce pressure sensors to be glued on the wings of an aircraft and on a satellite mockup to evaluate the performance of such systems in flight. 
There are more than 200 sensors per wing distributed into three profiles along the underside and the upper of the wings. These sensors are assembled in "gloves" compliant. The gloves are about 2-4 mm thick and 30-35 cm wide and there lengths vary according to location and density of sensors needed. The sensors must be identified, located and synchronized to reconstruct the profiles of pressure. The technological barriers identified are the access method to the medium, taking into account the high-data rate and the concurrent access especially when the frame in the application layer can reach 500 Mbps.

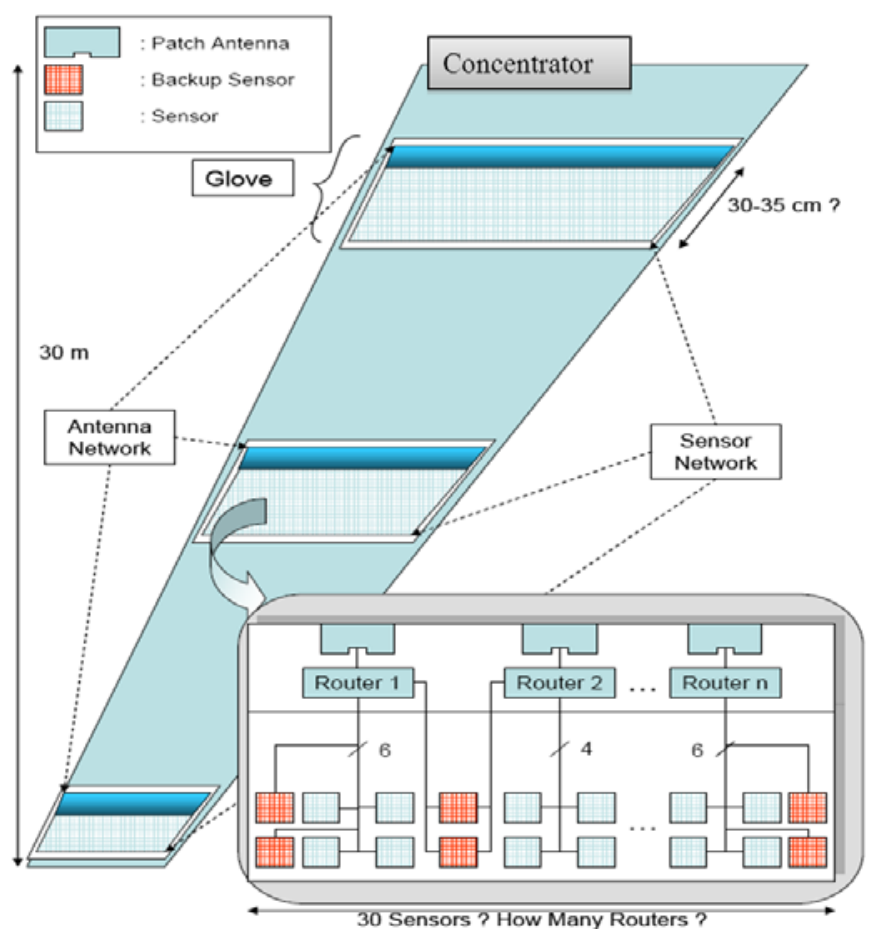

Figure 1: Wireless Sensor Network Architecture in the aircraft wings

The scenario, presented in figure 2 , is composed of a wireless node should communicate with 8 pressure sensitive sensors. A measurement is sampled at the rate of $22 \mathrm{KHz}$ and digitalized using 16 bits; the throughput provided is $341 \mathrm{Kbps}$ per sensor. Thus, the throughput at the node level is 8.17 Mbps.

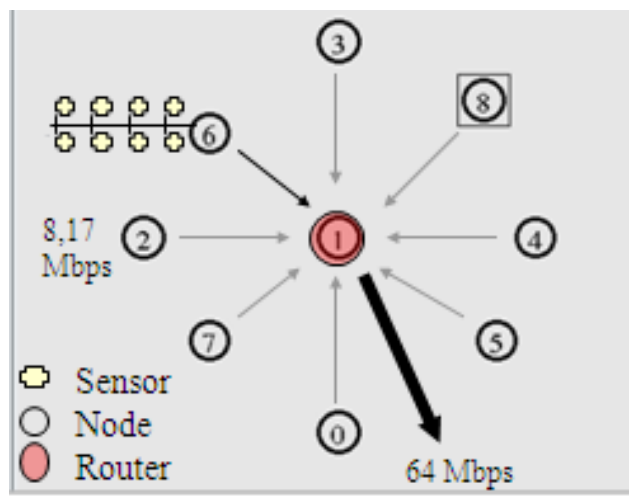

Figure 2: Scenario of the Wireless Sensor Network
Each sensor must be synchronized on the same time basis and all sensors must be synchronized together with a precision of $0.1^{\circ}$ phase shift from 2 to $256 \mathrm{~Hz}(139 \mu$ s to $1.08 \mu \mathrm{s})$ and at $1 \mu$ s for higher frequencies. Samples shall be dated with a precision of $+/-1 \mu$ s.

Nodes communicate with the router, which in its turn, communicates with 4 other routers scattered on the wing of the aircraft. The throughput at each router is 65,361 Mbps. At the last level the throughput at the concentrator level can exceed $400 \mathrm{Mbps}$. It is the gateway between the wireless network and industrial application. The downward flow is the transfer from the hub to the nodes. The upward flow is the transfer from the nodes to the hub.

Table 1 describes an example of some requirements needed by some wireless nodes and identifies the DataStream at each point.

Table 1: throughputs on each wireless node for spatial and aeronautic applications

\begin{tabular}{|l|l|l|l|l|l|}
\hline Application & Sensor & \multicolumn{4}{|c|}{ Throughput (kbps) } \\
\cline { 3 - 6 } & & sensor & node & router & concentrator \\
\hline Spatial & Pressure & 320 & 7660 & 47872 & $\mathbf{4 7 8 7 2}$ \\
\hline Aeronautics & Pressure & $\mathbf{3 4 1}$ & $\mathbf{8 1 7 0}$ & $\mathbf{6 5 3 6 1}$ & $\mathbf{3 9 2 1 6 4}$ \\
\hline
\end{tabular}

Indeed, the specifications of the communication system must take into account all constraints in order to accomplish the performance and the Quality of Service requirements: high data rate, minimum packet lost, minimum jitter, high availability and minimum delays have to be respected.

\section{OVERVIEW OF THE WIMEDIA-UWB COMMUNICATION}

The Wimedia Alliance has specified a physical layer standard based on Multiband Orthogonal Frequency Division Multiplexing (MB-OFDM) with a data rate of up to $480 \mathrm{Mbps}$ and a fully distributed Medium Access Control (MAC) protocol for UWB communication. Devices might use contention based Prioritized Channel Access (PCA) for asynchronous traffic and/or reservation based Distributed Reservation Protocol (DRP) for isochronous traffic.

\section{The UWB Physical Layer}

The protocol is designed for being a low-power with good facilities for QoS communication. UWB, shown in figure 3, operates over unlicensed 3.1 to $10.6 \mathrm{GHz}$ band, transferring at data rate from $54 \mathrm{Mbps}$ to $480 \mathrm{Mbps}$ at a limited transfer power of $-41.3 \mathrm{dBm} / \mathrm{Mhz}$.

UWB uses a PSK (Phase Shift Keying) simple binary based multiband-OFDM modulation technique for low rate communication, while for high rates DCM (Dual-carrier modulation) was used.

The MB-OFDM technique was first developed by IEEE 802.15.3a and standardized by ECMA [7] to be very similar to many conventional wireless OFDM systems. 


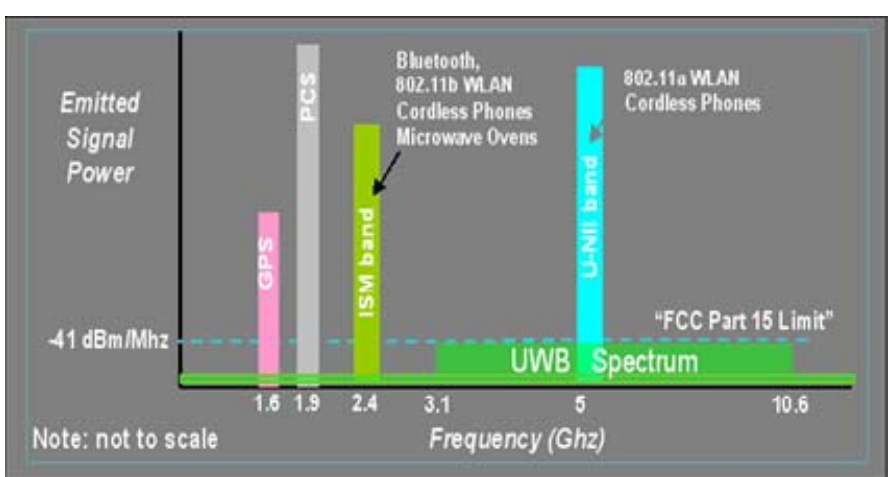

Figure 3: UWB’s spectrum usage

These rates are split in fourteen $528 \mathrm{MHz}$ band, grouped in five band groups. Data are encoded over 122 sub-carriers (100 data, 10 guards, 12 pilots). MB-OFDM uses frequency hopping sequence to send data packets into one single group

The most important among which is the capacity of capturing the preamble in dense multipath environments with a simple implementation. This advantage is one of the decisive points in the choice of MB-OFDM as the Wimedia UWB Physical Layer.

\section{The Wimedia MAC}

Wimedia Mac sub-Layer describes a fully distributed architecture which no central piconet is required to manage the communication between nodes. All devices have the same functionalities and each node sees itself as a central component and determines its neighbors by listening and communicating with them to coordinate the access the medium. All nodes share the bandwidth on the TDMA fashion. Thus, only one node can transmit data at a given time [9].

Wimedia uses a distributed approach to coordinate the channel access. Each node sees itself as a central component and determines its neighbors by listening and communicating with them to negotiate when each one can have a chance to access the medium. Wimedia radios share the notion of $65 \mathrm{~ms}$ long superframe (figure 4). A superframe is divided into 256 medium Access slots (MAS) and is composed of two parts: the first few MAS are called the beacon period and the other slots are called the data period. Devices use the beacon period to negotiate the priority to access the medium. The use of 'MAS', Wimedia allows several devices to cooperate with each other to share the medium access without requiring any master of the wireless bus to coordinate their transmission. This approach lets Wimedia support a high data rate communication up to 480 Mbps, and can be expected to move to gigabit data rates in the future. Devices use the Beacon Period to negotiate the medium access, schedule the time share and control the reservation of the superframe by each node. The Data Transfer Period is used to send and receive data. The DTP is divided into 2 periods: a contention based Prioritized Channel Control (PCA) for asynchronous traffic and/or reservation based Distributed Reservation Protocol (DRP) for isochronous traffic.

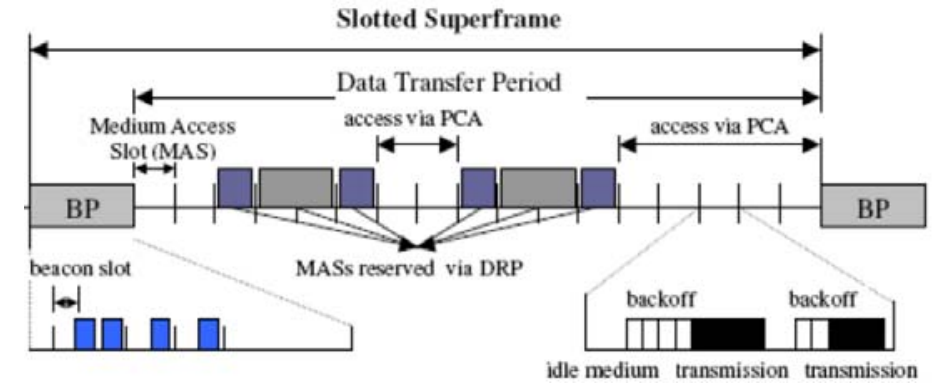

Figure 4: Wimedia MAC Superframe

\section{a. The beacon Period (BP)}

The BP is composed up to 32 MAS $^{1}, 85 \mu$ s each one. The two first slots are signaling slots. When a new node wants to join the network, it must verify that there is at least one available slot to be used to join the group. Node can join the network and synchronize itself with the other nodes in the some group, to form the Beacon Group to facilitate contention-free frame exchanges while exploring medium reuse over different spatial regions [8]. During the BP, each node sends an Information Element (IE) into its beacon packet to all other neighbors and receives all other beacon packet from them. The IEs include other information, like the IE DRP, which is necessary to the MAS reservation and determines the occupation of the superframe by each node during the Data Transfer Period. The BPOIE ${ }^{2}$ maintains a list of all neighbors using the same BPST ${ }^{3}$ in the same Beacon Group. Nodes can detect collisions in the last superframe and decreases the probability of the collision during the DTP by adapting there throughputs and the number of slots allowed.

\section{b. The Prioritized Channel Control (PCA)}

The PCA protocol is similar to the EDCA access method defined in the IEEE 802.11e draft standard. It is CSMA/CA based method with a selective back off priorities used in IEEE 802.11e. The difference between them lies in the use of UWBPHY. Coming from the low power generated by the UWBPHY (below to the noise signal), the carrier listening defined by the PCA protocol is not based on the energy detection, but it is preamble based listening. The channel is considered busy when a preamble is received, all nodes sense the carrier during the Arbitration Inter-Frame Space (AIFS) period, if the medium is available, node wishing to send listen during random several slots (in current specification the duration of a time slot is $8 \mu \mathrm{s}$ and the contention period can be specified by two lower and higher bounds like $[0, \mathrm{w}])$.

\section{c. Distributed Reservation Protocol (DRP)}

DRP is a free contention channel access, and it is used by nodes to negotiate and reserve bandwidth which the QoS contract established between devices. A reservation is defined by a subset of MASs during the BP. During the data transfer period only single device can access to the medium and allowed to send data. This kind of reservation is used to send

\footnotetext{
${ }^{1} 32$ is the maximum MAS allowed to the BP, but they can be less.

${ }^{2}$ Beacon Period Occupation Information

${ }^{3}$ Beacon Period Start Time
} 
isochronous traffic like in real time stream/media application. This protocol is useful for home WPAN meshed networks that require a high data rate, large bandwidth and low packet loss. To establish a reservation, a node must negotiate the data transfer period with its neighbors. There is no need of a central piconet between nodes, the Wimedia protocol is fully distributed and is a peer-to-peer like network.

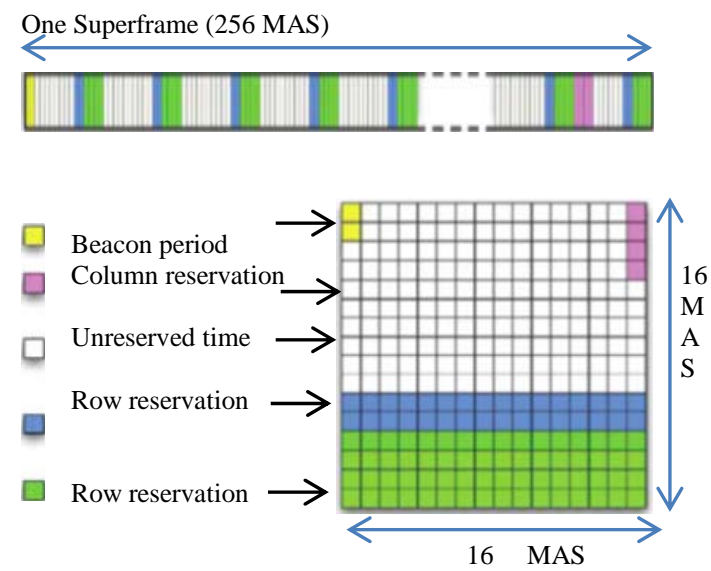

Figure 5: conversion of the superframe to bit map to allow DRP protocol

Figure 5 describes an example of bandwidth reservation in the DRP protocol. The first column describes the BP: during this period all transmitting nodes must occupy at least one slot. Like we described before, the super frame can be divided into 16 logical zones, the first zone can be considered like a beacon period, and other zones can be also considered as data transfer period. In figure 5, the superframe is mapped like a matrix to show how the DRP protocol works. In fact, the time slot reservation in line offers periodic slots to guarantee an isochronous traffic, while a column reservation is used to send burst traffic. For example, the two first row slots reserved during the 15 columns describe that a node transmitting data periodically during 2 MASs in each area. The four other lines describe the reservation of 4 MAS at each data zone. The top right 4 MAS refers to a node which reserves less bandwidth to send data only in the 15th zone [9]. The last described node reserves the lowest amount of bandwidth, while the first one reservation occupies the highest amount of the bandwidth. Nodes coordinate between each other without the use of central piconet to manage the communication; it is peer-to-peer like architecture. The DRP is well suited protocol for real time multimedia application.

\section{The convergence layer}

Wimedia differs from previous communication technologies in that it was designed to support several different styles of wireless communication simultaneously. The chosen approach aims to provide a convergence layer to be used by many technical communities as possible. Wireless USB (WUSB) was the first application to adopt the Wimedia protocol, and also a standard called WINET for running Internet protocol. Wireless 1394 and Wimedia Bluetooth has been adopted to support this new approach.

\section{a. Wireless USB:}

Certified Wireless USB is a specification for a wireless extension of standard USB by removing the cable and put in its place the UWB radio. The capacity and the data transfer rate can reach $480 \mathrm{Mbps}$ within a range of 3 to 10 meters.

WUSB uses the Wimedia-UWB technology to access the medium and it profits from all protocols defined above in the Wimedia protocol including the beaconing (discovery and control of reservation) and the DRP protocol. WUSB defines a channel encapsulated within the superframe which provides the data transfer over the DRP protocol. A channel is a continuous sequence of control packets called management commands (MMC Micro-scheduled Management Command), transmitted by the host during the MAC layer reservation. The MMCs contain necessary information to identify the host, control structures for the I/O and references temporal sequence to the next MMC. These links provide a set of spot recognized by the control nodes to enable them to join the group beacon provides equipment WUSB.

\section{b. WINET}

WINET known as IP over UWB [4] (also referred to as Wimedia IP) is a new generation of network communication broadband technology just released in 2007. The Wimedia IP includes the same functionalities possible over Ethernet: IP, Bridging (a bridge is like a Wi-Fi access point) and can be easily connected to Ethernet, Wi-Fi or IEEE 802.3.

Wimedia Alliance defined a Logical Link Control layer Networking Protocol (referred to as WLP) compliant with IEEE 802 standards. For example, a TCP/IP protocol stack designed for an IEEE 802.3 environment will also work with a Wimedia environment, using this protocol. In addition to support for straightforward application migration, this protocol also preserves data structures to facilitate the design of bridges between a Wimedia network and other IEEE 802 or compatible wired or wireless networks.

QoS is achieved by reserving fixed point-to-point streams allocated with the DRP protocol or by mapping IP traffic prioritization into the PCA protocol traffic level.

\section{MODEL OF THE WIMEDIA-UWB PROTOCOL}

Prior to any circuit development, simulation is used to perform the possibility of using a Wimedia compliant architecture. The simulation was made using NS-2.

\section{The NS-2 Protocol Stack}

This simulator does not provide a Wimedia-UWB Layer, so we developed the protocol stack presented in figure 6 . The UWB Physical Layer manages the transmission of bits over the antenna. 
The Wimedia Mac Sub-Layer provides all means and services for frame transmission between the physical layer and all other higher layers.

Beacons, Distributed Reservation Protocol (DRP) and Prioritized Contention Access (PCA) modules are chosen regarding the need of the protocol: the Beacon module is responsible for beaconing protocol and bitwise operator was used to select beacons used by each node; DRP and PCA manage the super frame reservation using both dynamic reservation and contention protocol.

High level layers are designed to support high rate communication and higher throughput.

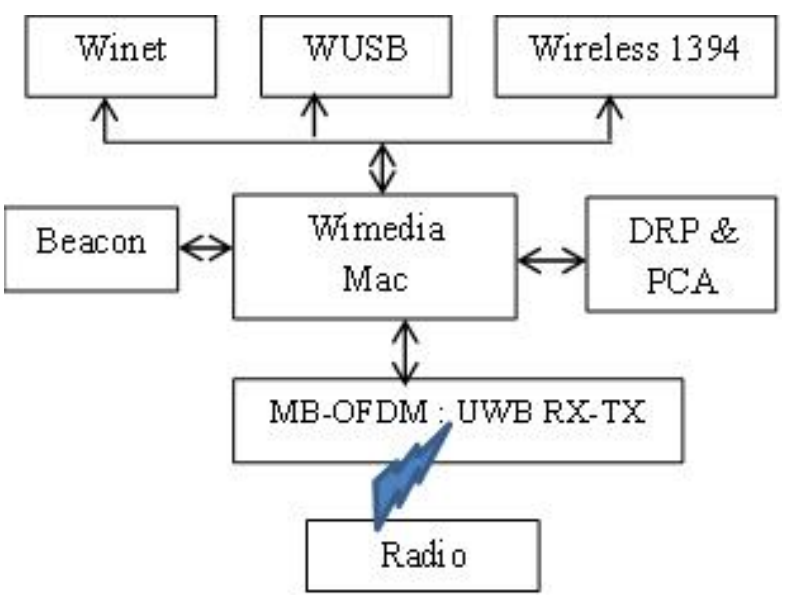

Figure 6: The model of Wimedia-UWB Layers

The class diagram presented in figure 7 points out the Physical Layer of the Wimedia protocol. The main class is the WimediaPhy which is used to manage all communication through the antenna. The techniques of Modulation implemented within the UWB communication module are both QPSK and DCM.

A New physical state was implemented within the Physical Layer called OFDM_PHY_STANDBY to model an advanced antenna state in order to support the management of the node hibernation and the detection of the preamble.

Because the physical layer modeled in the NS-2 simulator support only the power detection like these used in the for WiFi standards, an additional parameters has been added to this model in order to detect the preamble of the Wimedia frame.

The beacon protocol which manages the beaconing module was implemented within the Wimedia Mac Layer to support node discovering within the same beacon group. The purpose of this module is to facilitate the process of node discovering, the temporal synchronization between more superframes, the creation and sharing of the superframe and the reduction of interference. It offers an efficient way to provide the dynamic allocation of time slots within the superframe.

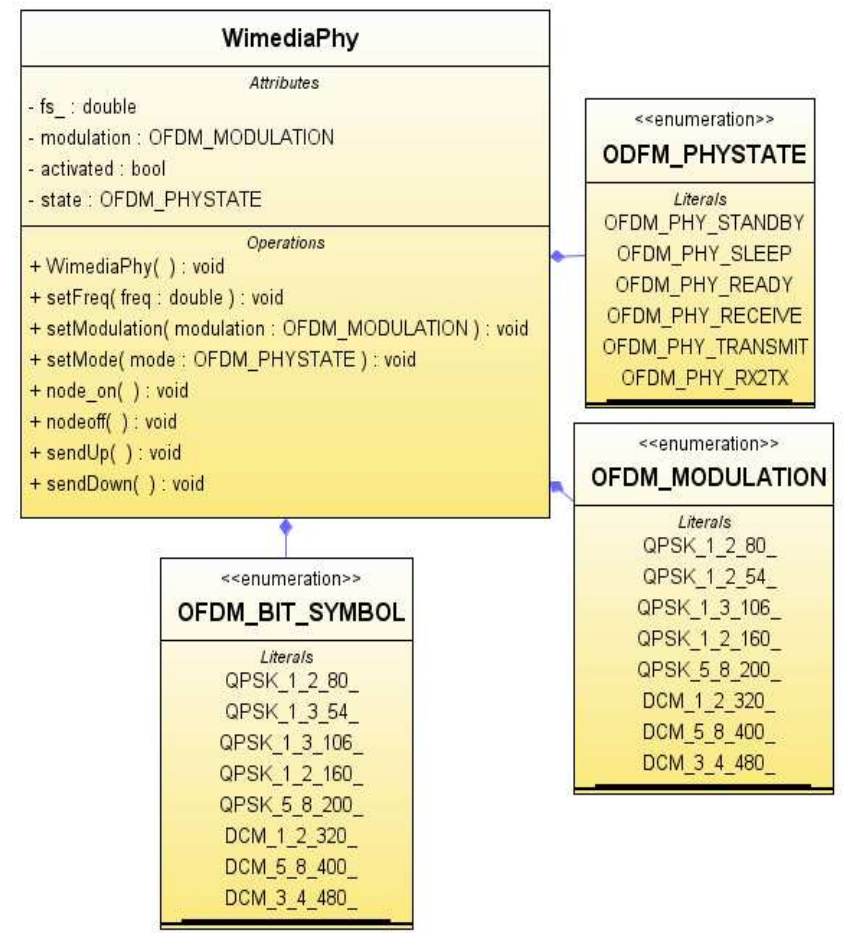

Figure 7: Wimedia Physical Layer (MB-OFDM) Class Diagram

Furthermore, the beacon module starts with a channel scan in order to discover all beacon time slots allowed within the Beacon Period Start Time (BPST) [10].

Then it schedules all received time slots to manage the dynamic reservation access to the superframe and allocates to each node the bandwidth it requires. The Beacon frame manages the beacon scheduling within the BPST to prevent superframe fragmentation and allowing the time slots reservation during the Data transfer Period (DTP).

Many options are added to this module to support the merging of beacons, the clustering and management of alien beacon groups. All these information were exchanged within the Information Elements (IEs).

To complete the protocol stack implementation, we designed a Wimedia MAC layer. It includes all necessary frame formats to support the PCA and DRP protocols. The Beacon frame aims to fix nodes within the same Beacon cluster, and then insure the exchange of the IEs required by the protocol.

The Beacon Frame manages the beacon scheduling within the BPST in order to avoid the superframe fragmentation and provide the time slots allocation needed during the data transfer period. The CTS and RTS frames serve for the backoff algorithm to manage the PCA protocol. UDR and UDA frames manage the DRP protocol.

\section{Model of the upper layers}

We have implemented a WUSB layer in order to provide a complete architecture to be used in spatial and Aeronautics projects. The WUSB like implementation describes the mandatory parts needed in our context: the packet type enumeration specifies the frame that a node sends during the sending/receiving process. The complete descriptions are given 
in [3]. The token packet is initialized to support different types of transactions (see the spec for more details).

This implementation was adopted to provide an adaptation layer between the Wimedia-UWB layers and the higher layers.

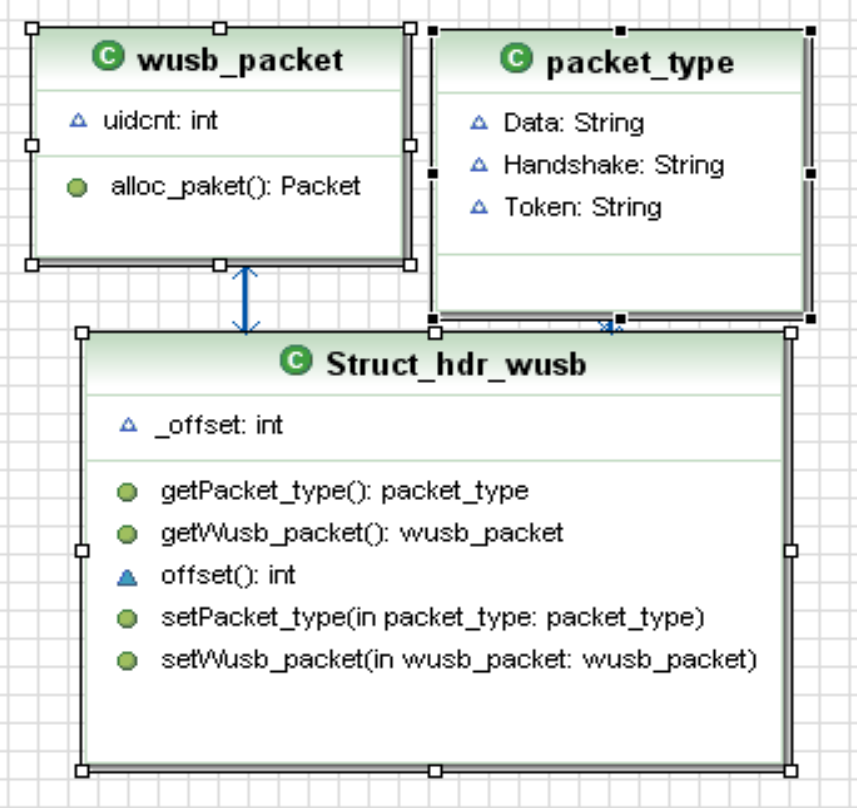

Figure 8: NS-2 WUSB Packet Implementation

We adopted the NS-2 representation of Agent to ensure the data communication between all nodes. So, we have represented all data transfer mechanism as an NS-2 Agent, like described in figure 8 . This is an easy method to communicate with high layer and to provide the interoperability with other Wimedia convergence layers.

Winet and Wireless IEEE 1394 were implemented, but only the WUSB protocol was used to evaluate the project. Their architecture is similar with some particularities attached to the specification of each protocol.

\section{EVALUATION WITH SIMULATIONS}

The purpose of the simulation is to evaluate the performance of the communication in Wireless Field Bus communication in aeronautic industry. These performances concern the throughput and the packet loss (Byte Error Rate).

To remind the network architecture studied in the simulation, the first scenario which has been studied, shown in figure 2, is composed of central point's, which communicate through wireless channels with 8 sensors; each node communicates with a real time sensor at $8,17 \mathrm{Mb} / \mathrm{s}$ throughput rate. Depending on the need of the designed application the throughputs can vary from 65,361 Mbps to $400 \mathrm{Mb} / \mathrm{s}$.

The delay between two generated packets from a desired node was 2 micro-seconds. The packet size was fixed at 4095 Bytes because we used the isochronous transfer mode of the Wimedia protocol. According to the bit rate that each node should generate we have varied this rate in order to determine the maximum rate that the router supports.
On the node side, the throughput that must be reached is 8.17 Mbps. The simulation results show that this data rate can be touched and can run over these limits.

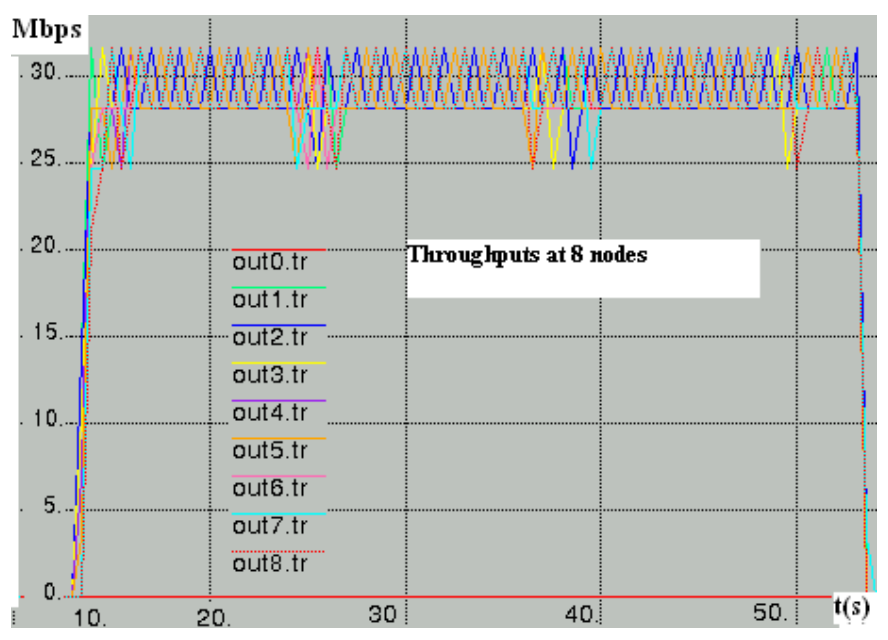

Figure 9: Throughputs in the nodes side

The second scenario that has been tested includes 8 routers. Each router receives data packets from 8 nodes. The throughputs at the routers sides are presented in figure 10 .

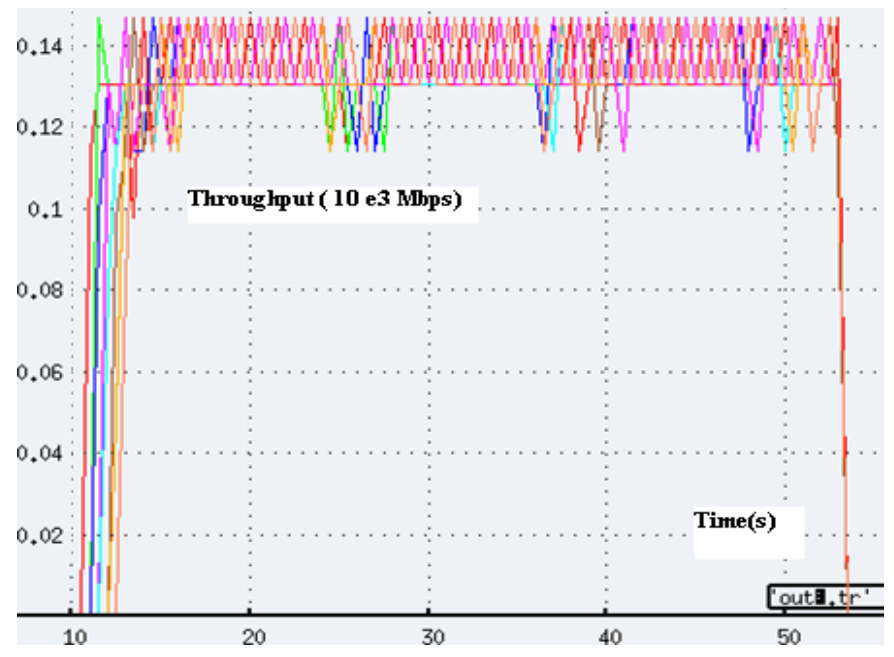

Figure 10 Throughput at router level

We conclude that the router can support the network traffic as the maximum throughput received by a Router is specified at $64 \mathrm{Mbps}$. In the simulator, the router can support at least 125Mbits/s.

In fact, the highest throughput value reached in the simulation was at $140 \mathrm{Mbps}$. The limit value of the simulation was attained at $125 \mathrm{Mbps}$. We increased the packet size during the simulation and we found that the throughput increases also. The Wimedia protocol envisages a large packet size (4095 Bytes) to conserve the isochronous transfer data mode implemented within USB 2.0 and IEEE 1394.

Figure 11 points out the progress of the throughput at different level of the network architecture. The throughput 
increases linearly with the packet size up to 125 Mbps. Then the throughput remains stable.

The linear increase of the curve proves that our protocol is more adapted for large packet sizes. The stagnation is abnormal and seems to be related to the saturation of the NS-2 Simulator, because the protocol was not completely designed.

However, the simulator limit (125 Mbps) is higher than the requested throughput for a single router simulation (64 Mbps).

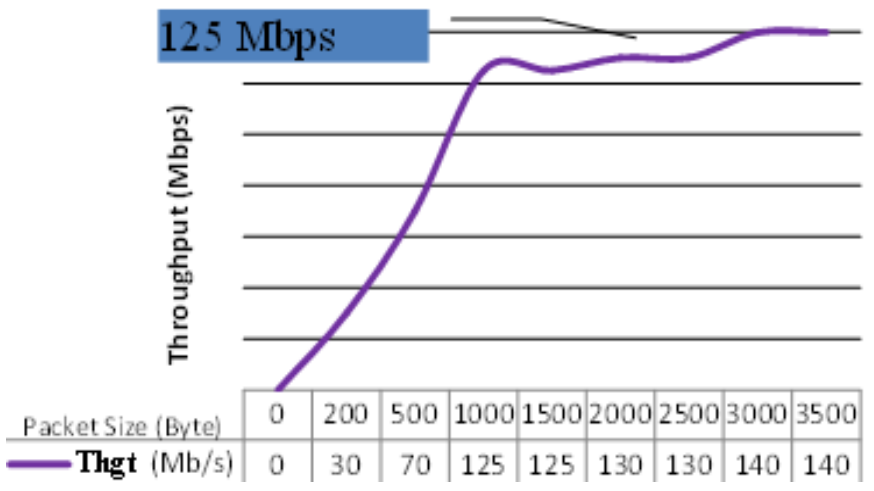

Figure 11: NS-2 Wimedia model Simulation

To improve the performance of the simulation when wireless nodes move, mobility was added to this third scenario. One master node was put in the central side, and other nodes moves around it. Nodes are slaves and only send data to the central point. The distance between all nodes is about 5 meters and nodes move at more than 10 meters.

The purpose was to generalize the approach studied to mobile networks. So, figure 12 shows the drawbacks of the developed Wimedia Mac protocol when devices become mobile. Indeed, the quality of information received at each node level was not very impressive. The majority of packets send by a slave mobile node are not received by the master node. The quality of service needed for the communication is not satisfied

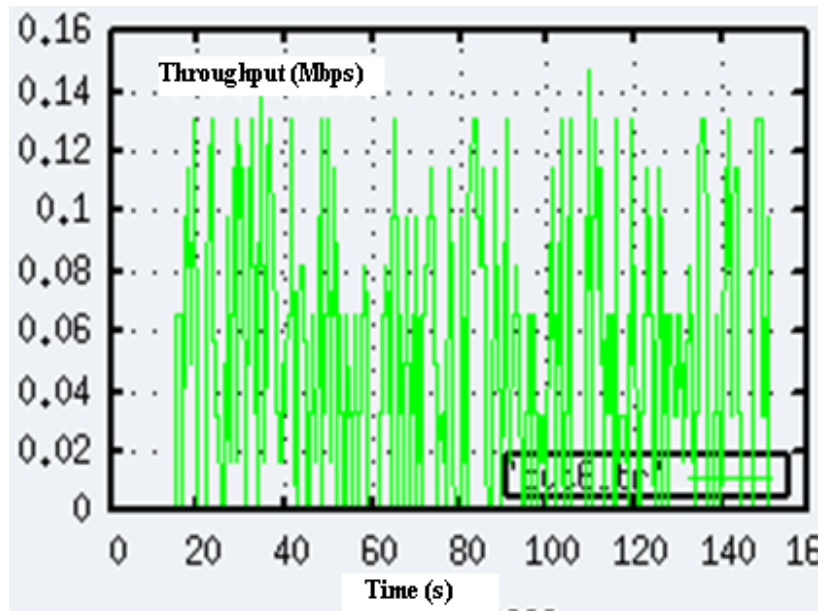

Figure 12 : The mobility effect on moving nodes
These simulation results will be confronted with measurements done in Hardware Testbed to verify the conformance of the simulation results with the experimentation.

\section{TEST AND MEASUREMENTS}

The simulation allowed the performance analyses of the requirements needed by the architecture. To evaluate a system's compliance with its specified requirements and confront the experimentation with the simulation, Hardware System Testing is conducted on integrated. UWB. The parameters used in the simulation model, like the distance between nodes, the parameters of the Wimedia Mac and the hopping sequence of the physical layer was injected to perform realistic measurements. These experiments need to be performed in the test bed are mandatory to valid the capacity of Wimedia system to fulfill our need, including the packet error rate and the throughputs.

The MB-OFDM signal used for the experiment is generated by a commercially available evaluation kit from Wisair [8].The DV9110M development provide MB-OFDM compliant modulation with three Wimedia-MBOA sub-bands, each of 528 $\mathrm{MHz}$ bandwidth in the band group 1 from $3.168 \mathrm{GHz}$ to 4.752 GHz. The average output power is UWB compliant with a value of $80 \mu \mathrm{W}(-41.3 \mathrm{dBm} / \mathrm{MHz})$.

The hopping sequence is set as $\mathrm{f} 1, \mathrm{f} 2 \mathrm{f} 3 \mathrm{fl}, \mathrm{f} 2, \mathrm{f} 3$, where $\mathrm{f} 1$ is the center frequency of the lower sub-band $(3.423 \mathrm{GHz})$, and $\mathrm{f} 2$ is the center frequencies of the middle $(3.960 \mathrm{GHz})$ and $\mathrm{f} 3$ is the upper sub-bands $(4.488 \mathrm{GHz}$ ).

Two Wisair cards at the master side to transmit the data and the slave side to receive the data. Many measurements were performed on Airbus wings and inside a Euro Star 3000 satellite.

We present in table 2 the packet error rate inside the satellite and on indoor airplane system. We evaluated the quality of the link by comparing the number of packets sent with the number of packets correctly received. This measurement corresponds to the number of Ethernet packets that has been sent by the transmitter but never received by the receiver: the packet error rate (PER).

To validate the propagation, the emitter and receiver are first placed in the same compartment of the satellite, and then they are placed indoor.

$\underline{\text { Table 2: Packet Error Lost indoor and inside satellite }}$

\begin{tabular}{|c|c|c|}
\hline \multirow{2}{*}{$\begin{array}{c}\text { Data rate } \\
\text { (Mbps) }\end{array}$} & \multicolumn{2}{|c|}{ Frame Error rate (\%) } \\
\cline { 2 - 3 } & Inside satellite & Indoor \\
\hline $\mathbf{5 3 . 3}$ & 86.2 & $<1$ \\
\hline $\mathbf{8 0}$ & 87.4 & $<1$ \\
\hline $\mathbf{1 0 6}$ & 88.2 & $<1$ \\
\hline $\mathbf{1 6 0}$ & 100 & $<1$ \\
\hline $\mathbf{2 0 0}$ & 100 & $<1$ \\
\hline $\mathbf{3 2 0}$ & 100 & $<1$ \\
\hline
\end{tabular}


Indeed, the quality of information received is so weak, due to metallic walls, that even beacons sent by the master cannot be interpreted by the slave.

In spite of many measurements done, it seems that the indoor measurements are more efficient regarding the inside satellite measurements. It seems to be related to the cavities and the metallic wall in the satellite mockup. Beacon send by the slave node seems to not be received at the master node. The master broadcast beacon frames to all considered slaves which use them to synchronize their own frames with other nodes.

Table 3 shows the influence of mobility on the throughput. It appears that the entire data rate is realizable below 5 meters. But, this limit is not achievable when the distance between nodes increases. It seems that the effect of this degradation is associated with the destructive interference due to the multipath scheme. In spite of the robustness of the MB-OFDM scheme, destructive interference could declines the Packet Error Rate. These experimental results confirm the simulation results when nodes become mobile.

Table 3 : Packet Error Lost caused by the node mobility

\begin{tabular}{|c|c|c|c|c|c|}
\hline $\begin{array}{c}\text { Data rate } \\
\text { (Mbps) }\end{array}$ & \multicolumn{5}{|c|}{ Packet Error rate (\%) } \\
\cline { 2 - 6 } & $1 \mathrm{~m}$ & $2 \mathrm{~m}$ & $5 \mathrm{~m}$ & $10 \mathrm{~m}$ & $15 \mathrm{~m}$ \\
\hline 53.3 & 0 & 0 & 0 & 6.2 & 2.23 \\
\hline 80 & 0 & 0 & 0 & 11.9 & 5.51 \\
\hline 106 & 0 & 0 & 0.1 & 36.1 & 8.5 \\
\hline 160 & 0 & 0 & 0.6 & 60 & 100 \\
\hline
\end{tabular}

\section{CONCLUSION}

In this paper we present a new simulation model for the Wimedia protocol for the NS-2 framework. The simulation helps us to validate our main intention to use Wimedia protocol for rapid prototyping wireless field buses in spatial and aeronautic industries. We have performed software simulation using the NS-2 Simulator, to evaluate the features of the designed Wimedia-Uwb layer in spatial and aeronautic systems. The simulation proves that the requirements of the communication system are satisfied.

These simulations were completed with measurements directly in real Hardware System (test bed, aircraft and satellite). The considerations implemented within the simulation model were used as parameters in the Testbed to validate the compliance with the standard. We observe that MB-OFDM is very suitable for fixed indoor communication. But tests performed in the satellite mockup and when nodes move the behavior is not satisfying the requirements. A limitation of simulating wireless sensor networks with NS-2 resides in the physical layer simulation.
A prospect of this work is to extend ns-2 simulator to include more physical layer parameters in the simulation and possible investigation have to be done further.

Future works are considering the implementation of this protocol in a FPGA or ASIC devices to be used and deployed in a real Aeronautics system and inside the satellite.

\section{REFERENCES}

[1] Mandke, K., Haewoon, N., Lasya, Y., \& Christian, Z. (May 6, 2003). The Evolution of UWB and IEEE 802.15.3a for Very High Data Rate WPAN. EE 381K-11 Wireless Communications UWB Group, The University of Texas at Austin .

[2] Retrieved from Wimedia Alliance: http://www.wimedia.org/en/index.asp

[3] Wireless Universal Serial Bus Specification Revision 1.0 released May 12, 2005 . (2008, 6). Retrieved from ttp://www.usb.org/developers/wusb/

[4] Wimedia Logical Link Control Protocol. Wlp Specification: Approved Draft 1.0. (07, August 13). from http://www.wimedia.org/en/index.asp

[5] Bluetooth Special Interest Group: https://www.bluetooth.org/

[6] Batra, A., Balakrishnan, J. \& Dabak, A., 2004. Multi-band OFDM: a new approach for UWB. In Circuits and Systems, 2004. ISCAS '04. Proceedings of the 2004. International Symposium on. pp. 365-368 Vol.5.

[7] ECMA, 2008. High Rate Ultra Wideband PHY and MAC Standard, ECMA international. Available at: http://www.ecmainternational.org/publications/files/ECMAST/ECMA368.pdf

[8] Wisair, 2009. Commercial Website. Available at: http://www.wisair.com/ [Accessed March 23, 2009].

[9] Pavon, Jd.P. Sai Shankar N Gaddam, V. Challapali, K. Chun-Ting Chou. The MBOA-WiMedia Specification for Ultra Wideband Distributed Networks. Toronto, Ont., Canada, IEEE Communication Magazine, June 2006

[10] Vishnevsky, V.M. Lyakhov, A.I. Safonov, A.A. Mo, S.S. Gelman, A.D. Study of Beaconing in Multihop Wireless PAN with Distributed Control. IEEE Mobile Communication. Russian Acad. of Sci., Moscow; 